PROCEEDINGS OF THE

AMERICAN MATHEMATICAL SOCIETY

Volume 140, Number 6, June 2012, Pages 2127-2140

S 0002-9939(2011)11216-4

Article electronically published on October 21, 2011

\title{
ISOMETRIES OF THE UNITARY GROUP
}

\author{
OSAMU HATORI AND LAJOS MOLNÁR \\ (Communicated by Richard Rochberg)
}

\begin{abstract}
In this paper we describe all surjective isometries of the unitary group of a complex Hilbert space. A result on Thompson isometries of the space of all invertible positive elements of a unital $C^{*}$-algebra is also presented.
\end{abstract}

\section{INTRODUCTION}

The classical Mazur-Ulam theorem states that every surjective isometry $T$ between normed real-linear spaces is necessarily affine, and hence it equals a surjective linear isometry followed by a translation. The main point of the proof of that important result is to show that the isometry $T$ under consideration respects the operation of the arithmetic mean in the sense that

$$
T\left(\frac{x+y}{2}\right)=\frac{T x+T y}{2}
$$

holds for every pair $x, y$ of points in the domain of $T$. Having proved this property and using continuity, it follows immediately that $T$ is affine. The starting point of our investigations is the miraculous short proof of the Mazur-Ulam theorem given by Väisälä in [12].

In the recent paper 2 we have managed to develop further the basic ideas of Väisälä to noncommutative settings and presented several results concerning the algebraic behavior of isometries on general groups. In fact, our results show that under various conditions, the isometries under consideration locally preserve the socalled inverted Jordan triple product $b a^{-1} b$, an operation that takes over the role played by the arithmetic mean in the classical Mazur-Ulam theorem. Furthermore, in 2 we have utilized the obtained general results to describe certain isometries (or, more generally, so-called $d$-preserving transformations) of various groups of continuous functions on compact Hausdorff spaces. In the present paper we continue our study and apply the general results in 2 to determine the structure of isometries

Received by the editors February 12, 2011.

2010 Mathematics Subject Classification. Primary 47B49.

Key words and phrases. Unitary group, Hilbert space, isometry, Mazur-Ulam theorem, $C^{*}$ algebra, Thompson metric.

The first author was partly supported by the Grants-in-Aid for Scientific Research, Japan Society for the Promotion of Science.

The second author was supported by the Alexander von Humboldt Foundation (Germany), by the Hungarian Scientific Research Fund (OTKA) K81166 NK81402, and by the TÁMOP 4.2.1./B-09/1/KONV-2010-0007 project implemented through the New Hungary Development Plan co-financed by the European Social Fund and the European Regional Development Fund.

(C)2011 American Mathematical Society Reverts to public domain 28 years from publication 
of a particularly important noncommutative metric group, the unitary group on a Hilbert space.

To make the presentation complete, in what follows we recall the necessary definitions and briefly summarize the results in 2] that we shall need in the proofs below. We remark that in 2] we have considered so-called $d$-preserving transformations, and hence the definitions and results are somewhat more general there, but here we present the corresponding material only for metrics and isometries.

First fix the following. In Definitions 1 to $3,(X, d)$ denotes a metric space, where $X$ is a nonempty subset of a group $G$ with the property that

$$
y x^{-1} y \in X \text { holds for every pair } x, y \in X \text {. }
$$

Definition 1 (Condition $\mathrm{B}(\cdot, \cdot))$. Let $a, b \in X$. We say that $\mathrm{B}(a, b)$ holds for $(X, d)$ if the following are fulfilled:

(B1) For all $x, y \in X$ we have

$$
d\left(b x^{-1} b, b y^{-1} b\right)=d(x, y)
$$

(B2) There exists a constant $K>1$ such that we have

$$
\begin{gathered}
\qquad d\left(b x^{-1} b, x\right) \geq K d(x, b) \\
\text { for all } x \in L_{a, b}=\left\{x \in X: d(a, x)=d\left(b a^{-1} b, x\right)=d(a, b)\right\} .
\end{gathered}
$$

Definition 2 (Condition $\left.\mathrm{C}_{1}(\cdot, \cdot)\right)$. Let $a, b \in X$. We say that $\mathrm{C}_{1}(a, b)$ holds for $(X, d)$ if the following are fulfilled:

(C1) For every $x \in X$ we have $a x^{-1} b, b x^{-1} a \in X$.

(C2) The equality $d\left(a x^{-1} b, a y^{-1} b\right)=d(x, y)$ holds for every pair $x, y \in X$.

Definition 3 (Condition $\left.\mathrm{C}_{2}(\cdot, \cdot)\right)$. Let $a, b \in X$. We say that $\mathrm{C}_{2}(a, b)$ holds for $(X, d)$ if there exists a $c \in X$ with $c a^{-1} c=b$ such that the equality $d\left(c x^{-1} c, c y^{-1} c\right)$ $=d(x, y)$ holds for every pair $x, y \in X$.

Concerning the previous condition observe the following. Assume that $X$ is 2divisible; i.e. it has the property that for every $x \in X$ there is a $y \in X$ such that $y^{2}=x$. Let $a, b \in X$. We assert that there exists $c \in X$ such that $c a^{-1} c=b$. To see this, take $d \in X$ with $d^{2}=a$, and $f \in X$ with $f^{2}=d b^{-1} d$. Setting $c=d f^{-1} d$ we have $c \in X$ and

$$
c a^{-1} c=d f^{-1} d a^{-1} d f^{-1} d=d\left(f^{-1}\right)^{2} d=b .
$$

Above are the conditions under which we obtained the following results in [2] concerning the local algebraic behavior of isometries of groups.

Let us fix the following. In Propositions 4, 5 we suppose that for $i=1,2,\left(X_{i}, d_{i}\right)$ is a metric space, $X_{i}$ is a nonempty subset of a group $G_{i}$ and $y x^{-1} y \in X_{i}$ holds for all $x, y \in X_{i}$. Let $\phi: X_{1} \rightarrow X_{2}$ be a surjective isometry.

Proposition 4. Pick $a, b \in X_{1}$. Suppose that $\mathrm{B}(a, b)$ holds for $\left(X_{1}, d_{1}\right)$ and $\mathrm{C}_{1}\left(\phi(a), \phi\left(b a^{-1} b\right)\right)$ holds for $\left(X_{2}, d_{2}\right)$. Then we have

$$
\phi\left(b a^{-1} b\right)=\phi(b)(\phi(a))^{-1} \phi(b) .
$$

For the next result we agree that $X_{2}$ is said to be 2-torsion free if the unit element $e$ of $G_{2}$ belongs to $X_{2}$ and for any $x \in X_{2}$ the equality $x^{2}=e$ implies $x=e$. 
Proposition 5. Pick $a, b \in X_{1}$. Suppose that $\mathrm{B}(a, b)$ holds for $\left(X_{1}, d_{1}\right)$ and $\mathrm{C}_{2}\left(\phi(a), \phi\left(b a^{-1} b\right)\right)$ holds for $\left(X_{2}, d_{2}\right)$. If $X_{2}$ is 2-divisible and 2-torsion free, then we have

$$
\phi\left(b a^{-1} b\right)=\phi(b)(\phi(a))^{-1} \phi(b) .
$$

\section{NEW RESUlTS AND PROOFS}

In this section we use the above results from 2 to describe the isometries of the unitary group of a Hilbert space. In fact, our first result concerns the isometries of subgroups of the isometry group $\operatorname{Iso}(\mathcal{B})$ of any complex Banach space $\mathcal{B}$.

We denote by $\operatorname{Iso}(\mathcal{B})$ the group of all surjective linear isometries of $\mathcal{B}$. Clearly, Iso $(\mathcal{B})$ is a closed multiplicative group in the Banach algebra $B(\mathcal{B})$ of all bounded linear operators on $\mathcal{B}$ equipped with the usual operator norm $\|\cdot\|$. The structure of the isometry group that reflects the geometrical structure of the underlying Banach space is extensively studied in the literature both from algebraic and analytical aspects. In fact, Iso $(\mathcal{B})$ is one of the most important linear algebraic groups. Below we contribute to that study by pointing out certain algebraic properties of the surjective isometries of $\operatorname{Iso}(\mathcal{B})$. In particular, in the next result we consider isometries of an arbitrary subgroup of $\operatorname{Iso}(\mathcal{B})$ while in the main result of the paper we determine the general form of all surjective isometries of the unitary group of a Hilbert space.

Theorem 6. Let $\mathcal{B}$ be a complex Banach space and $\mathfrak{G}$ be a subgroup of $\operatorname{Iso}(\mathcal{B})$ equipped with the metric $d$ coming from the operator norm. Suppose that $\phi: \mathfrak{G} \rightarrow \mathfrak{G}$ is a surjective isometry. Then we have

$$
\phi\left(V U^{-1} V\right)=\phi(V)(\phi(U))^{-1} \phi(V)
$$

for all $U, V \in \mathfrak{G}$ that satisfy $d(U, V)<1 / 2$.

Proof. Since the metric $d$ on $\operatorname{Iso}(\mathcal{B})$ is inverse and translation invariant, it is clear that $\mathrm{C}_{1}\left(\phi(U), \phi\left(V U^{-1} V\right)\right)$ holds whenever $U, V \in \mathfrak{G}$.

Let $U, V \in \mathfrak{G}$ be such that $d(U, V)<1 / 2$. We show that $\mathrm{B}(U, V)$ also holds and then Proposition 4 applies. In fact, the condition (B1) in Definition 1 is apparently satisfied. It remains to check (B2). We assert that with $K=2-2 d(U, V)>1$, the inequality

$$
d\left(V W^{-1} V, W\right) \geq K d(W, V)
$$

holds for every $W \in L_{U, V}$, where

$$
L_{U, V}=\left\{W \in \mathfrak{G}: d(U, W)=d\left(V U^{-1} V, W\right)=d(U, V)\right\} .
$$

To see this, let $W \in L_{U, V}$. Then we see that

$$
d(W, V) \leq d(W, U)+d(U, V)=2 d(U, V)<1,
$$

and since $V^{-1}$ is a surjective isometry, we compute

$$
\frac{1}{2}>\left\|\frac{W+V}{2}-V\right\|=\left\|\frac{W+V}{2} V^{-1}-I\right\|,
$$

where $I$ is the identity operator on $\mathcal{B}$. Since $B(\mathcal{B})$ is a Banach algebra, we obtain that $((W+V) / 2) V^{-1}$ is an invertible element in $B(\mathcal{B})$ and so is $W+V$. We claim 
that $\left\|(W+V)^{-1} z\right\|_{\mathcal{B}} \leq 1$ holds for every $z \in \mathcal{B}$ with $\|z\|_{\mathcal{B}}=K$. Indeed, for such a vector $z \in \mathcal{B}$ set $y=(W+V)^{-1} z$. Then we compute

$$
\begin{aligned}
K & =\|z\|_{\mathcal{B}}=\|(W+V) y\|_{\mathcal{B}}=\|2 W y+(V-W) y\|_{\mathcal{B}} \\
& \geq 2\|y\|_{\mathcal{B}}-\|V-W\|\|y\|_{\mathcal{B}} \\
& \geq 2\|y\|_{\mathcal{B}}-2 d(U, V)\|y\|_{\mathcal{B}}=K\|y\|_{\mathcal{B}},
\end{aligned}
$$

so $\|y\|_{\mathcal{B}} \leq 1$ holds. Since $\left(W(W+V)^{-1}\right)^{-1}=I+V W^{-1}$, we deduce that

$$
\left\|\left(V W^{-1}+I\right)^{-1} x\right\|_{\mathcal{B}}=\left\|W(W+V)^{-1} x\right\|_{\mathcal{B}}=\left\|(W+V)^{-1} x\right\|_{\mathcal{B}}
$$

holds for every $x \in \mathcal{B}$. Thus, $\left\|\left(V W^{-1}+I\right)^{-1} x\right\|_{\mathcal{B}} \leq 1$ if $\|x\|_{\mathcal{B}}=K$. Therefore, we compute

$$
\begin{aligned}
K d(W, V) & =K\|V-W\|=K\left\|V W^{-1}-I\right\| \\
& =\sup _{x \in \mathcal{B},\|x\|_{\mathcal{B}}=K}\left\|\left(V W^{-1}-I\right) x\right\|_{\mathcal{B}} \\
& =\sup _{x \in \mathcal{B},\|x\|_{\mathcal{B}}=K}\left\|\left(V W^{-1}-I\right)\left(V W^{-1}+I\right)\left(V W^{-1}+I\right)^{-1} x\right\|_{\mathcal{B}} \\
& \leq \sup _{y \in \mathcal{B},\|y\|_{\mathcal{B}} \leq 1}\left\|\left(V W^{-1}-I\right)\left(V W^{-1}+I\right) y\right\|_{\mathcal{B}} \\
& =\left\|\left(V W^{-1}-I\right)\left(V W^{-1}+I\right)\right\| \\
& =\left\|V W^{-1} V W^{-1}-I\right\|=\left\|V W^{-1} V-W\right\|=d\left(V W^{-1} V, W\right) .
\end{aligned}
$$

This gives us that (B2) holds, too. Applying Proposition 4 we have

$$
\phi\left(V U^{-1} V\right)=\phi(V)(\phi(U))^{-1} \phi(V)
$$

for all $U, V \in \mathfrak{G}$ with $d(U, V)<1 / 2$.

We note that the assumption $d(U, V)<1 / 2$ in Theorem 6 cannot be omitted. To see this, let $Y$ be a compact Hausdorff space with at least two points and denote by $C(Y)$ the Banach space of all the complex-valued continuous functions on $Y$. Let $\mathfrak{G}$ be the group of all composition operators of the form $T_{\eta}(u)=u \circ \eta(u \in C(Y))$ with any self-homeomorphism $\eta$ of $Y$. (Observe that $\mathfrak{G}$ is exactly the automorphism group of $C(Y)$.) Using Urysohn's lemma it is easy to check that

$$
d\left(T_{\mu}, T_{\nu}\right)= \begin{cases}0, & \text { if } \mu=\nu, \\ 2, & \text { otherwise }\end{cases}
$$

holds for any pair $\mu, \nu$ of homeomorphisms of $Y$. Consequently, every bijective transformation of $\mathfrak{G}$ onto itself is a surjective isometry.

Theorem 6 says that any surjective isometry of the isometry group of any complex Banach space has the property that it preserves the inverted Jordan triple product of close enough elements. The following technical lemma will enable us to obtain from this local preserver property the corresponding global preserver property in the case of Hilbert spaces.

Lemma 7. For $i=1,2$, let $G_{i}$ be a group and $X_{i}$ a nonempty subset of $G_{i}$ such that $y x^{-1} y \in X_{i}$ holds for every pair $x, y \in X_{i}$. Suppose that $\phi: X_{1} \rightarrow X_{2}$ is a map, $n$ is a positive integer, and $\left\{a_{k}\right\}_{k=0}^{2^{n}}$ is a finite sequence in $X_{1}$ such that we have $a_{k+1} a_{k}^{-1} a_{k+1}=a_{k+2}$ and

$$
\phi\left(a_{k+1} a_{k}^{-1} a_{k+1}\right)=\phi\left(a_{k+1}\right)\left(\phi\left(a_{k}\right)\right)^{-1} \phi\left(a_{k+1}\right)
$$


for all $0 \leq k \leq 2^{n}-2$. Then it follows that $a_{2^{n-1}} a_{0}^{-1} a_{2^{n-1}}=a_{2^{n}}$ and

$$
\phi\left(a_{2^{n-1}} a_{0}^{-1} a_{2^{n-1}}\right)=\phi\left(a_{2^{n-1}}\right)\left(\phi\left(a_{0}\right)\right)^{-1} \phi\left(a_{2^{n-1}}\right) .
$$

Proof. For a given positive integer $n$ and any sequence $\left\{a_{k}\right\}_{k=0}^{2^{n}}$ in $X_{1}$, denote by $A(n)$ that we have $a_{k+1} a_{k}^{-1} a_{k+1}=a_{k+2}$ and

$$
\phi\left(a_{k+1} a_{k}^{-1} a_{k+1}\right)=\phi\left(a_{k+1}\right)\left(\phi\left(a_{k}\right)\right)^{-1} \phi\left(a_{k+1}\right)
$$

for all $0 \leq k \leq 2^{n}-2$. Similarly, let us denote by $C(n)$ that $a_{2^{n-1}} a_{0}^{-1} a_{2^{n-1}}=a_{2^{n}}$ and

$$
\phi\left(a_{2^{n-1}} a_{0}^{-1} a_{2^{n-1}}\right)=\phi\left(a_{2^{n-1}}\right)\left(\phi\left(a_{0}\right)\right)^{-1} \phi\left(a_{2^{n-1}}\right)
$$

hold. We prove the implication $A(n) \Longrightarrow C(n)$ by induction on $n$. This is obvious for $n=1$. Assume now that it is true that "if $A(n-1)$ is satisfied for a finite sequence of $2^{n-1}+1$ terms, then $C(n-1)$ also holds for that sequence". We will show that this implies that "if $A(n)$ is satisfied for a sequence of $2^{n}+1$ terms, then $C(n)$ also holds for that sequence". Suppose $A(n)$ is satisfied for the sequence $\left\{a_{k}\right\}_{k=0}^{2^{n}}$ in $X_{1}$. We first show that for every $k=0, \ldots, 2^{n}-4$,

$$
a_{k+2} a_{k}^{-1} a_{k+2}=a_{k+4}
$$

and

$$
\phi\left(a_{k+2} a_{k}^{-1} a_{k+2}\right)=\phi\left(a_{k+2}\right)\left(\phi\left(a_{k}\right)\right)^{-1} \phi\left(a_{k+2}\right)
$$

hold. Let $0 \leq k \leq 2^{n}-4$. Then

$$
\begin{aligned}
a_{k+4} & =a_{k+3} a_{k+2}^{-1} a_{k+3} \\
& =\left(a_{k+2} a_{k+1}^{-1} a_{k+2}\right) a_{k+2}^{-1}\left(a_{k+2} a_{k+1}^{-1} a_{k+2}\right)=a_{k+2} a_{k+1}^{-1} a_{k+2} a_{k+1}^{-1} a_{k+2} \\
& =a_{k+2} a_{k+1}^{-1}\left(a_{k+1} a_{k}^{-1} a_{k+1}\right) a_{k+1}^{-1} a_{k+2}=a_{k+2} a_{k}^{-1} a_{k+2},
\end{aligned}
$$

and hence (2) holds. We also see that

$$
\begin{aligned}
\phi\left(a_{k+2} a_{k}^{-1} a_{k+2}\right) & =\phi\left(a_{k+3} a_{k+2}^{-1} a_{k+3}\right)=\phi\left(a_{k+3}\right)\left(\phi\left(a_{k+2}\right)\right)^{-1} \phi\left(a_{k+3}\right) \\
& =\phi\left(a_{k+2} a_{k+1}^{-1} a_{k+2}\right)\left(\phi\left(a_{k+2}\right)\right)^{-1} \phi\left(a_{k+2} a_{k+1}^{-1} a_{k+2}\right) \\
& =\phi\left(a_{k+2}\right)\left(\phi\left(a_{k+1}\right)\right)^{-1} \phi\left(a_{k+2}\right)\left(\phi\left(a_{k+1}\right)\right)^{-1} \phi\left(a_{k+2}\right) \\
& =\phi\left(a_{k+2}\right)\left(\phi\left(a_{k+1}\right)\right)^{-1} \phi\left(a_{k+1} a_{k}^{-1} a_{k+1}\right)\left(\phi\left(a_{k+1}\right)\right)^{-1} \phi\left(a_{k+2}\right) \\
& =\phi\left(a_{k+2}\right)\left(\phi\left(a_{k}\right)\right)^{-1} \phi\left(a_{k+2}\right),
\end{aligned}
$$

and hence (3) holds.

Next, letting $b_{k}=a_{2 k}$ for $0 \leq k \leq 2^{n-1}$, the sequence $\left\{b_{k}\right\}_{k=0}^{2^{n-1}}$ satisfies $A(n-1)$ due to (2) and (3). By our assumption we infer that $C(n-1)$ holds for $\left\{b_{k}\right\}_{k=0}^{2^{n-1}}$, i.e. $b_{2^{n-2}} b_{0}^{-1} b_{2^{n-2}}=b_{2^{n-1}}$ and

$$
\phi\left(b_{2^{n-2}} b_{0}^{-1} b_{2^{n-2}}\right)=\phi\left(b_{2^{n-2}}\right)\left(\phi\left(b_{0}\right)\right)^{-1} \phi\left(b_{2^{n-2}}\right) .
$$

For $b_{0}=a_{0}$ and $b_{2^{n-2}}=a_{2^{n-1}}$, we obtain that $C(n)$ holds for $\left\{a_{k}\right\}_{k=0}^{2^{n}}$.

In the main result of the paper that follows we describe the structure of all surjective isometries of the unitary group of a complex Hilbert space. To formulate the result we need the following notation. Let $H$ be an arbitrary complex Hilbert space. Beside the adjoint operation on the algebra $B(H)$ of all bounded linear operators on $H$ we shall also need the operation of transposition. It is defined by choosing a complete orthonormal system (Hilbert basis) in $H$ and, for any operator 
$A$, considering the operator $A^{T}$ whose matrix in the given basis is the transpose of the corresponding matrix of $A$. It can be seen that the map $A \mapsto A^{T}$ is a well-defined linear *-antiautomorphism of $B(H)$. The conjugate $\bar{A}$ of an operator $A \in B(H)$ is defined by the formula $\bar{A}=A^{* T}$. After this preparation the result reads as follows.

Theorem 8. Let $H$ be a complex Hilbert space and denote by $\mathrm{U}(H)$ the group of all unitary operators on $H$ equipped with the metric that comes from the operator norm. Suppose that $\phi: \mathrm{U}(H) \rightarrow \mathrm{U}(H)$ is a surjective isometry. Then there exist unitaries $V, W \in \mathrm{U}(H)$ such that $\phi$ is of one of the following forms:

(U1) $\phi(A)=V A W^{*} \quad$ for all $A \in \mathrm{U}(H)$,

(U2) $\phi(A)=V A^{*} W^{*}$ for all $A \in \mathrm{U}(H)$,

(U3) $\phi(A)=V A^{T} W^{*}$ for all $A \in \mathrm{U}(H)$,

(U4) $\phi(A)=V \bar{A} W^{*} \quad$ for all $A \in \mathrm{U}(H)$.

Proof. We first note that every transformation of any of the forms (1) - (4) with some unitaries $V, W \in \mathrm{U}(H)$ is a surjective isometry of the unitary group. The content of the theorem is that the reverse statement is also true, any surjective isometry of $\mathrm{U}(H)$ is necessarily of one of those simple forms.

To begin the proof, first observe that the case $\operatorname{dim} H=1$ is rather trivial; hence we assume $\operatorname{dim} H \geq 2$. We claim that

$$
\phi\left(B A^{-1} B\right)=\phi(B) \phi\left(A^{-1}\right) \phi(B)
$$

holds for every pair $A, B \in \mathrm{U}(H)$. Pick $A, B \in \mathrm{U}(H)$. Since $A^{-1} B$ is unitary, there exists a selfadjoint operator $C \in B(H)$ such that $\exp i C=A^{-1} B$. Let $m$ be a positive integer such that $\exp \left(\|i C\| / 2^{m}\right)-1<1 / 2$. Then

$$
\left\|\exp \frac{i C}{2^{m}}-I\right\| \leq \exp \frac{\|i C\|}{2^{m}}-1<\frac{1}{2}
$$

holds. Let

$$
A_{k}=A \exp \frac{i k C}{2^{m}}
$$

for all $k=0,1,2, \ldots, 2^{m+1}$. Then we have $A_{0}=A, A_{2^{m}}=B$, and $A_{2^{m+1}}=$ $B A^{-1} B$. It is easy to check that

$$
A_{k+1}\left(A_{k}\right)^{-1} A_{k+1}=A_{k+2}
$$

holds for every $k=0,1,2, \ldots, 2^{m+1}-2$. We also have

$$
\left\|A_{k+1}-A_{k}\right\|=\left\|\exp \frac{i C}{2^{m}}-I\right\|
$$

for any $k=0,1,2, \ldots, 2^{m+1}-1$ since $A \exp \left(i k C / 2^{m}\right)$ is a unitary operator. Then by Theorem 6 it follows that

$$
\phi\left(A_{k+1}\left(A_{k}\right)^{-1} A_{k+1}\right)=\phi\left(A_{k+1}\right)\left(\phi\left(A_{k}\right)\right)^{-1} \phi\left(A_{k+1}\right)
$$

holds for every $k=0,1,2, \ldots, 2^{m+1}-2$. Applying Lemma 7 we deduce that

$$
\begin{aligned}
\phi\left(B A^{-1} B\right)=\phi\left(A_{2^{m}}\left(A_{0}\right)^{-1} A_{2^{m}}\right)=\phi\left(A_{2^{m}}\right)\left(\phi\left(A_{0}\right)\right)^{-1} \phi\left(A_{2^{m}}\right) & \\
& =\phi(B)(\phi(A))^{-1} \phi(B),
\end{aligned}
$$

and this gives the equality (4). 
Denoting $\phi_{0}(\cdot)=(\phi(I))^{-1} \phi(\cdot)$ it requires only an elementary calculation to check that $\phi_{0}$ is a bijective Jordan triple map on $\mathrm{U}(H)$; i.e. it satisfies

$$
\phi_{0}(B A B)=\phi_{0}(B) \phi_{0}(A) \phi_{0}(B)
$$

for all $A, B \in \mathrm{U}(H)$. Consequently, there is no serious loss of generality in assuming that the given surjective isometry $\phi$ on $\mathrm{U}(H)$ is unital (i.e. $\phi(I)=I)$ ) and satisfies the equality

$$
\phi(A B A)=\phi(A) \phi(B) \phi(A)
$$

for all $A, B \in \mathrm{U}(H)$.

In what follows we call a unitary operator $S \in \mathrm{U}(H)$ a symmetry if $S^{2}=I$. It requires only an easy algebraic argument to verify that $S \in \mathrm{U}(H)$ is a symmetry if and only if $S=I-2 P$ holds with some projection (selfadjoint idempotent) $P$ on $H$.

We assert that $\phi$ preserves the symmetries in both directions, meaning that $S \in \mathrm{U}(H)$ is a symmetry if and only if $\phi(S) \in \mathrm{U}(H)$ is a symmetry. Indeed, for any $S \in \mathrm{U}(H)$ we have

$$
S^{2}=I \Leftrightarrow S I S=I \Leftrightarrow \phi(S) \phi(I) \phi(S)=\phi(I) \Leftrightarrow \phi(S)^{2}=I .
$$

Pick any $U \in \mathrm{U}(H)$ and a symmetry $S$. Using $S^{2}=I, \phi(S)^{2}=I$ we infer that

$$
U S=S U \Leftrightarrow S U S=U \Leftrightarrow \phi(S) \phi(U) \phi(S)=\phi(U) \Leftrightarrow \phi(U) \phi(S)=\phi(S) \phi(U) .
$$

We claim that $\phi$ sends the scalar multiples of the identity to unitaries of the same kind. To see this, let $\lambda$ be a complex number of modulus one. As $\lambda I$ is a unitary operator which commutes with every symmetry, it follows from (6) that $\phi(\lambda I)$ is a unitary operator which also commutes with every symmetry. We have already learned that the symmetries are exactly those elements of $\mathrm{U}(H)$ which are of the form $I-2 P$ with some projection $P$. Therefore, we obtain that $\phi(\lambda I)$ commutes with every projection. By the spectral theorem, the closed linear hull of the set of all projections on $H$ equals $B(H)$. Hence we obtain that $\phi(\lambda I)$ commutes with every operator on $H$. This implies that $\phi(\lambda I)$ is a scalar multiple of the identity; i.e. we have a complex number $f(\lambda)$ of modulus one such that $\phi(\lambda I)=f(\lambda) I$. Clearly, $f(1)=1$ and the function $f: \mathbb{T} \rightarrow \mathbb{T}$ is an isometry. It follows that either we have $f(\lambda)=\lambda$ for all $\lambda \in \mathbb{T}$ or we have $f(\lambda)=\bar{\lambda}$ for all $\lambda \in \mathbb{T}$. In the former case we see that $\phi$ leaves the scalar multiples of the identity invariant. In the latter case we consider the transformation $(\phi(\cdot))^{*}$ which is a surjective isometry on $\mathrm{U}(H)$, it is a unital Jordan triple map and, in addition, it fixes the scalar multiples of the identity. Therefore, there is no loss of generality in assuming that $\phi(\lambda I)=\lambda I$ holds for all $\lambda \in \mathbb{T}$.

We next define a transformation on the set $\mathrm{P}(H)$ of all projections on $H$. Namely, for any $P \in \mathrm{P}(H)$, let

$$
\psi(P)=\frac{I-\phi(I-2 P)}{2} .
$$

By the structure of symmetries, it is easy to see that $\psi$ is a bijective map of $\mathrm{P}(H)$ onto itself. Moreover, we have that $\psi(0)=0, \psi(I)=I$ (these follow from $\phi(I)=I$ and $\phi(-I)=-I$, respectively) and that $\psi$ preserves commutativity in both directions (meaning that for any pair $P, Q \in \mathrm{P}(H)$ of projections, $P Q=Q P$ holds if and only if $\psi(P) \psi(Q)=\psi(Q) \psi(P))$. This latter assertion follows from (6). 
We now need a characterization of projections of rank one or corank one. Let us call such projections simple. For any subset $\mathcal{P}$ of $\mathrm{P}(H)$, denote by $\mathcal{P}^{k}$ the set of all elements of $\mathrm{P}(H)$ which commute with every element of $\mathcal{P}$. The characterization we need reads as follows: a nontrivial projection $P$ (meaning that $P \neq 0, I$ ) is simple if and only if for every projection $Q$ which commutes with $P$ we have that the set $\left(\{P, Q\}^{k}\right)^{k}$ has at most $2^{3}=8$ elements. The proof of this statement is given in [9], pp. 276-277. Since the bijective transformation $\psi$ on $\mathrm{P}(H)$ preserves commutativity in both directions and it fixes the trivial projections, we see that $\psi$ maps the set of all simple projections onto itself.

It is easy to see that for any pair $P, Q \in \mathrm{P}(H)$ of unitarily equivalent projections there is a finite sequence $R_{1}, \ldots, R_{n}$ of projections such that $P=R_{1}, R_{n}=Q$ and $\left\|R_{l}-R_{l+1}\right\|<1, l=1, \ldots, n-1$. In fact, this follows from the path-connectedness of the unitary group. As $\psi$ is isometric, we obtain that $\left\|\psi\left(R_{l}\right)-\psi\left(R_{l+1}\right)\right\|<1$, $l=1, \ldots, n-1$. It is a folk result that if the distance (in the operator norm) between two projections is less than 1, then they are unitarily equivalent. (Indeed, if $p, q$ are projections in any unital $C^{*}$-algebra such that $\|p-q\|<1$, then $s=$ $\left(1-(p-q)^{2}\right)^{-1 / 2}(p+q-1)$ can be checked to be a symmetry satisfying $\left.s p s=q.\right)$ Therefore, we obtain that $\psi\left(R_{l}\right)$ and $\psi\left(R_{l+1}\right), l=1, \ldots, n-1$ are all unitarily equivalent which gives us the unitary equivalence of $\psi(P)$ and $\psi(Q)$. As the inverse of $\psi$ is also an isometry of $\mathrm{P}(H)$, the reverse implication holds too, meaning that the unitary equivalence of $\psi(P)$ and $\psi(Q)$ implies that of $P$ and $Q$.

The above observations imply that there are two possible cases:

a) $\psi$ maps the set of all rank-one projections bijectively onto itself;

b) $\psi$ maps the set of all rank-one projections bijectively onto the set of all corank-one projections.

Let us first consider the case a); that is, assume that $\psi$ is a surjective isometry of the space $\mathrm{P}_{1}(H)$ of all rank-one projections on $H$. For any $P, Q \in \mathrm{P}_{1}(H)$ the possibly nonzero eigenvalues of $P-Q$ are $\pm \sqrt{1-\operatorname{tr} P Q}$ (here tr denotes the usual trace functional); see, e.g., 7, p. 127. Hence $\|P-Q\|=\sqrt{1-\operatorname{tr} P Q}$, and as $\psi$ is an isometry, it follows that

$$
\operatorname{tr} \psi(P) \psi(Q)=\operatorname{tr} P Q
$$

holds for every pair $P, Q$ of rank-one projections. Transformations possessing this property are important for their applications in quantum theory. Indeed, in the mathematical foundations of quantum mechanics, the pure states of a quantum system are represented by the rank-one projections on some corresponding Hilbert space (this is just one of the possible representations; there are others as well). The quantity tr $P Q$ is called the transition probability between the states represented by $P$ and $Q$. Bijective transformations on $\mathrm{P}_{1}(H)$ which preserve this quantity in the sense seen in (7) are called quantum mechanical symmetry transformations. By a celebrated theorem of Wigner, the structure of all such transformations is known. In fact, every quantum mechanical symmetry transformation is implemented by either a unitary or an antiunitary operator; i.e. it is of the form $P \mapsto U P U^{*}$, where $U$ is a unitary or antiunitary operator on $H$ (see, e.g., 7, p. 12). An antiunitary operator means a bijective conjugate-linear isometry on $H$. To avoid bothering with conjugate-linear operators, observe that if $U$ is antiunitary, then the map $A \mapsto\left(U A^{*} U^{*}\right)^{T}$ is a linear *-algebra automorphism of $B(H)$. It is well known that all such automorphisms are inner; i.e. they are implemented by unitary operators. 
This means that there is a unitary operator $V$ such that $\left(U A^{*} U^{*}\right)^{T}=V A V^{*}$ holds for all $A \in B(H)$. Inserting any rank-one projection $P$ in the place of $A$, we see that $U P U^{*}=\left(V P V^{*}\right)^{T}=V^{T^{*}} P^{T} V^{T}$. Therefore, we conclude that $\psi$, just as any quantum mechanical symmetry transformation, is either of the form $\psi(P)=$ $W P W^{*}$ for all $P \in \mathrm{P}_{1}(H)$ or of the form $\psi(P)=W P^{T} W^{*}$ for all $P \in \mathrm{P}_{1}(H)$ with some unitary operator $W$.

In the former case consider the transformation $A \mapsto W^{*} \phi(A) W$ on $\mathrm{U}(H)$, while in the latter case consider the transformation $A \mapsto W^{T} \phi(A)^{T} W^{* T}$ on $\mathrm{U}(H)$. Clearly, in both cases we obtain a unital Jordan triple map of $\mathrm{U}(H)$ which is a surjective isometry fixing all scalar multiples of the identity. Moreover, in addition to those, the new transformation fixes every so-called simple symmetry, which means any operator of the form $I-2 P$ with $P \in \mathrm{P}_{1}(H)$. Therefore, we may and do assume that our original transformation $\phi$ has all these properties.

Pick any projection $E$ and let $E^{\prime}$ be a projection such that $\phi(I-2 E)=I-2 E^{\prime}$. We show that $E^{\prime}=E$ or $E^{\prime}=I-E$. To see this, let $P$ be any rank-one projection. Using (6) we have

$$
\begin{aligned}
& P E=E P \Leftrightarrow(I-2 P)(I-2 E)=(I-2 E)(I-2 P) \\
& \Leftrightarrow \phi(I-2 P) \phi(I-2 E)=\phi(I-2 E) \phi(I-2 P) \\
& \quad \Leftrightarrow(I-2 P)\left(I-2 E^{\prime}\right)=\left(I-2 E^{\prime}\right)(I-2 P) \Leftrightarrow P E^{\prime}=E^{\prime} P .
\end{aligned}
$$

This means that $E, E^{\prime}$ commute with exactly the same rank-one projections. It is easy to see that a projection commutes with a rank-one projection if and only if the range of the rank-one projection in question is either orthogonal to, or included in, the range of the other projection. Therefore, we have that each vector from the range of $E$ is either in the range of $E^{\prime}$ or in its orthogonal complement. It is a quite known fact (and easy to check) that if a linear subspace of a linear space is included in the union of two linear subspaces, then it is necessarily included in one of them. Therefore, we deduce that the range of $E$ is included either in the range of $E^{\prime}$ or in its orthogonal complement. Interchanging the roles of $E$ and $E^{\prime}$ we obtain that either $E=E^{\prime}$ or $E=I-E^{\prime}$. It follows that either we have $\phi(I-2 E)=I-2 E$ or we have $\phi(I-2 E)=-(I-2 E)$. This means that for each symmetry $S$ we have $\phi(S)= \pm S$.

Pick an arbitrary unitary operator $U \in \mathrm{U}(H)$. It follows from (6) that for any symmetry $S \in \mathrm{U}(H), S$ commutes with $U$ if and only if $S= \pm \phi(S)$ commutes with $\phi(U)$. Consequently, for any projection $E$ we have that $E$ is in the commutant of $U$ (the set of all operators that commute with $U$ ) if and only if it is in the commutant of $\phi(U)$. The commutant of $U$ is a von Neumann algebra. Indeed, the fact that it is closed under taking adjoints is a consequence of the famous theorem of Fuglede stating that if a normal operator $N$ commutes with any operator $A$, then $N$ commutes with $A^{*}$, too. Any von Neumann algebra is the closed linear hull of the set of its projections. It then follows that the commutant of $U$ equals the commutant of $\phi(U)$ and hence the same holds for the double commutants (i.e. for the commutants of the commutants), too. Pick any projection $P \in \mathrm{P}(H)$ and different complex numbers $\lambda, \mu \in \mathbb{T}, \lambda \neq \mu$. The double commutant of $\lambda P+\mu(I-P)$ is the set of all operators of the form $\alpha P+\beta(I-P)$, where $\alpha, \beta$ are arbitrary complex numbers. The sketch of the proof of this assertion is as follows. Let $P$ be a projection on $H$. Since an operator $A$ commutes with the normal operator $\lambda P+\mu(I-P)$ if and only if $A$ commutes with its spectral resolution $\{P, I-P\}$, 
it follows that the commutant (resp. double commutant) of $\lambda P+\mu(I-P)$ equals the commutant (resp. double commutant) of $P$. Clearly, the operators of the form $\alpha P+\beta(I-P)$ with arbitrary complex numbers $\alpha, \beta$ are in the double commutant of $P$. Conversely, if $A$ belongs to the double commutant of $P$, we see that $A$ commutes with every rank-one subprojection of $P$ or $I-P$. Commuting with a rank-one projection implies that the range of the rank-one projection is an eigensubspace of $A$. Therefore, we obtain that the ranges of $P, I-P$ are invariant subspaces of $A$ and the restriction of $A$ onto any of those subspaces has the property that every nonzero vector from its domain is an eigenvector. This gives us that on the range of $P$ (resp. on that of $I-P), A$ is a scalar multiple of the identity. This yields that $A$ is of the form $A=\alpha P+\beta(I-P)$ with some complex numbers $\alpha, \beta$.

To proceed with the proof of the theorem, pick an arbitrary projection $P$ different from $I$. For every $\lambda \in \mathbb{T}$, the unitary operator $\phi(\lambda P+(I-P))$ belongs to its double commutant which is equal to the double commutant of $\lambda P+(I-P)$. Therefore, we have functions $f, g: \mathbb{T} \rightarrow \mathbb{T}$ such that

$$
\phi(\lambda P+(I-P))=f(\lambda) P+g(\lambda)(I-P)
$$

for all $\lambda \in \mathbb{T}$. As $\phi$ is a unital Jordan triple map, it follows easily that $f, g$ are multiplicative. Moreover, as $\phi$ is an isometry, we have

$$
\max \{|f(\lambda)-f(\mu)|,|g(\lambda)-g(\mu)|\}=|\lambda-\mu|
$$

for all $\lambda, \mu \in \mathbb{T}$. In particular, $f, g$ are continuous endomorphisms, i.e. characters of the circle group $\mathbb{T}$. It is a fundamental fact that then $f, g$ are necessarily power functions (functions of the form $z \mapsto z^{n}$ with some integer power $n$ ). See, for example, pp. 12-13 in Rudin's book [10]. As $|f(\lambda)-f(\mu)|,|g(\lambda)-g(\mu)| \leq|\lambda-\mu|$ for every $\lambda, \mu \in \mathbb{T}$, it follows that the powers can only be $-1,0,1$. Denote by $i d, \mathbf{1}$ the identity function and the identically 1 function on $\mathbb{T}$, respectively. We learn that $f, g \in\{i d, \mathbf{1}, \overline{i d}\}$.

Let us assume for a moment that $P$ is of rank one. Putting $\lambda=-1$ into (8), it follows from $\phi(I-2 P)=I-2 P$ that $g(-1)=1$. Consequently, for any rank-one $P$, the function $g$ is identically 1 on $\mathbb{T}$ and hence we have

$$
\phi(\lambda P+(I-P))=f(\lambda) P+(I-P)
$$

for every $\lambda \in \mathbb{T}$. As $\phi(\mu I)=\mu I$, computing the distance between $\lambda P+(I-P)$ and $\mu I$, and then the distance between their images $f(\lambda) P+(I-P)$ and $\mu I$, by the isometric property of $\phi$ we get

$$
\max \{|\lambda-\mu|,|1-\mu|\}=\max \{|f(\lambda)-\mu|,|1-\mu|\}
$$

for all $\lambda, \mu \in \mathbb{T}$. One can check very easily that from the three possibilities $f=$ $i d, \mathbf{1}, \overline{i d}$ only $f=i d$ satisfies the above equality. Therefore, we have

$$
\phi(\lambda P+(I-P))=\lambda P+(I-P)
$$

for all rank-one projections $P$ and complex numbers $\lambda \in \mathbb{T}$. We next assert that the same formula holds also for every projection $P$ different from $I$ regardless of its rank. Pick any $I \neq P \in \mathrm{P}(H)$ of rank at least two. We already know that

$$
\phi(\lambda P+(I-P))=f(\lambda) P+g(\lambda)(I-P), \quad \lambda \in \mathbb{T},
$$

holds with certain functions $f, g \in\{i d, \mathbf{1}, \overline{i d}\}$. Take a rank-one subprojection $Q$ of $P$. For any $\lambda, \mu, \nu \in \mathbb{T}$, consider the distances from $\lambda P+(I-P)$ to $\mu Q+(I-Q)$ and to $\nu I$, respectively. Then consider the distances among the corresponding images, 
that is, from $f(\lambda) P+g(\lambda)(I-P)$ to $\mu Q+(I-Q)$ and to $\nu I$, respectively. By the isometric property of $\phi$ we obtain the following equalities:

$$
\begin{gathered}
\max \{|\lambda-\mu|,|\lambda-1|\}=\max \{|f(\lambda)-\mu|,|f(\lambda)-1|,|g(\lambda)-1|\}, \\
\max \{|\lambda-\nu|,|1-\nu|\}=\max \{|f(\lambda)-\nu|,|g(\lambda)-\nu|\}
\end{gathered}
$$

for all $\lambda, \mu, \nu \in \mathbb{T}$. It requires very simple considerations to check that from all of the nine possibilities $f, g \in\{i d, \mathbf{1}, \overline{i d}\}$ only one can really occur, namely, $f=i d$ and $g=1$. Therefore, we obtain that

$$
\phi(\lambda P+(I-P))=\lambda P+(I-P)
$$

holds for all projections $P$ and complex numbers $\lambda \in \mathbb{T}$.

As $\phi$ is a unital Jordan triple map, we see that

$$
\begin{aligned}
& \phi\left(U_{n} U_{n-1} \ldots U_{1} U_{1} \ldots U_{n-1} U_{n}\right) \\
& \quad=\phi\left(U_{n}\right) \phi\left(U_{n-1}\right) \ldots \phi\left(U_{1}\right) \phi\left(U_{1}\right) \ldots \phi\left(U_{n-1}\right) \phi\left(U_{n}\right)
\end{aligned}
$$

holds for any finite sequence $U_{1}, \ldots, U_{n}$ of unitary operators. Putting $U_{l}=\lambda_{l} P_{l}+$ $\left(I-P_{l}\right), l=1, \ldots, n$, where $P_{1}, \ldots, P_{n}$ are pairwise orthogonal projections with sum $I$, we obtain

$$
\phi\left(\sum_{l=1}^{n} \lambda_{l}^{2} P_{l}\right)=\sum_{l=1}^{n} \lambda_{l}^{2} P_{l} .
$$

By the spectral theorem, the operators of the form $\sum_{l=1}^{n} \lambda_{l}^{2} P_{l}$ are dense in $\mathrm{U}(H)$. Therefore, $\phi$ fixes the elements of a dense subset of $\mathrm{U}(H)$, which plainly implies that $\phi$ acts as the identity on the whole group $\mathrm{U}(H)$. This is what we could conclude in the case a).

As for the case b), using similar arguments as above, we show that this in fact cannot occur if $\operatorname{dim} H \geq 3$ (in the two-dimensional case, a) and b) are the same). So assume that $\operatorname{dim} H \geq 3$ and $\psi$ maps the set of all rank-one projections bijectively onto the set of all corank-one projections. It follows that the transformation $P \mapsto$ $I-\psi(P)$ is a quantum mechanical symmetry transformation for which Wigner's theorem applies. We deduce that there is a unitary operator $W$ such that $\psi$ is either of the form $\psi(P)=W(I-P) W^{*}$ for all $P \in \mathrm{P}_{1}(H)$ or of the form $\psi(P)=$ $W(I-P)^{T} W^{*}$ for all $P \in \mathrm{P}_{1}(H)$. In the former case consider the transformation $A \mapsto W^{*} \phi(A) W$ on $\mathrm{U}(H)$, while in the latter case consider the transformation $A \mapsto W^{T} \phi(A)^{T} W^{* T}$ on $\mathrm{U}(H)$. Clearly, in both cases we obtain a unital Jordan triple map of $\mathrm{U}(H)$, which is a surjective isometry fixing all scalar multiples of the identity. Moreover, the new transformation has the property that it maps every symmetry of the form $I-2 P, P$ being a rank-one projection, to its negative, i.e. we have $\phi(I-2 P)=-(I-2 P)$. In what follows we denote the new transformation by the same symbol $\phi$.

We can proceed just as in the case a) and verify that for any projection $E$ we have either $\phi(I-2 E)=I-2 E$ or $\phi(I-2 E)=-(I-2 E)$. This means that for every symmetry $S$, we have $\phi(S)= \pm S$. One can continue with showing that the commutants and hence also the double commutants of $U$ and $\phi(U)$ coincide. From this one can infer that for any projection $P$ different from $I$ there exist functions $f, g: \mathbb{T} \rightarrow \mathbb{T}$ such that

$$
\phi(\lambda P+(I-P))=f(\lambda) P+g(\lambda)(I-P)
$$


holds for every $\lambda \in \mathbb{T}$. Just as in the case a) we obtain that $f, g \in\{i d, \mathbf{1}, \overline{i d}\}$. If $P$ is of rank one, then we know that $\phi(I-2 P)=-(I-2 P)$. This implies that $f(-1)=1$, which gives us that $f$ is identically 1 on $\mathbb{T}$. Examining the distances between $\lambda P+(I-P)$ and $\mu I$, and then between the images $P+g(\lambda)(I-P)$ and $\mu I$, for all $\lambda, \mu \in \mathbb{T}$ we easily conclude that from the three possibilities $g=i d, \mathbf{1}, \overline{i d}$ only $g=i d$ can occur. It then follows that

$$
\phi(\lambda P+(I-P))=P+\lambda(I-P)
$$

for all rank-one projections $P$ and complex numbers $\lambda \in \mathbb{T}$. Let $P$ now be a rank-two projection. We know that there are functions $f, g \in\{i d, \mathbf{1}, \overline{i d}\}$ such that

$$
\phi(\lambda P+(I-P))=f(\lambda) P+g(\lambda)(I-P)
$$

holds for every $\lambda \in \mathbb{T}$. Pick a rank-one subprojection $Q$ of $P$, and for all $\lambda, \mu, \nu \in \mathbb{T}$ examine the distances from $\lambda P+(I-P)$ to $\mu Q+(I-Q)$ and to $\nu I$, respectively, and also examine the distances among the corresponding images. By the isometric property of $\phi$ we obtain the following equalities:

$$
\begin{gathered}
\max \{|\lambda-\mu|,|\lambda-1|\}=\max \{|f(\lambda)-1|,|f(\lambda)-\mu|,|g(\lambda)-\mu|\}, \\
\max \{|\lambda-\nu|,|1-\nu|\}=\max \{|f(\lambda)-\nu|,|g(\lambda)-\nu|\}
\end{gathered}
$$

for all $\lambda, \mu, \nu \in \mathbb{T}$. It is easy to check that from the nine possibilities $f, g \in\{i d, \mathbf{1}, \overline{i d}\}$ none of them can really happen. Hence we have arrived at a contradiction, verifying that the case b) cannot occur if $\operatorname{dim} H \geq 3$.

To summarize the content of the main steps of the proof, we can now tell that after multiplying our original transformation $\phi$ by a unitary operator from the left, and (if necessary) composing by the adjoint operation, and (if necessary) composing by the transposition operation and, finally, multiplying by some unitary operator from the right and by its adjoint from the left, we necessarily obtain the identity transformation on $\mathrm{U}(H)$. But this is exactly what is formulated in the theorem; hence the proof is complete.

We note that norm isometries between open subgroups of invertible elements in Banach algebras have been studied by the first author in [1. In the remaining part of the paper we consider another important metric on another noncommutative structure appearing in the theory of operator algebras and its isometries. It is the Thompson metric on the set of all invertible positive elements in a unital $C^{*}$-algebra.

Let $\mathcal{A}$ be a unital $C^{*}$-algebra and denote by $\mathcal{A}_{+}^{-1}$ the set of all invertible positive elements in $\mathcal{A}$, which is clearly a subset of the multiplicative group $\mathcal{A}^{-1}$ of all invertible elements in $\mathcal{A}$. The so-called Thompson metric on $\mathcal{A}_{+}^{-1}$ is defined by

$$
d_{T}(x, y)=\log \max \{\inf \{t>0: x \leq t y\}, \inf \{t>0: y \leq t x\}\}
$$

for any $x, y \in \mathcal{A}_{+}^{-1}$. (Here $\leq$ denotes the usual order among the selfadjoint elements of $\mathcal{A}$.) In fact, this definition works in a much more general setting; see [11. It is not difficult to verify that for any $x, y \in \mathcal{A}_{+}^{-1}$ we have

$$
d_{T}(x, y)=\left\|\log x^{-\frac{1}{2}} y x^{-\frac{1}{2}}\right\|
$$

(see, e.g., 6]). It is known that this metric coincides with the geodesic distance corresponding to the natural Finsler geometrical structure of $\mathcal{A}_{+}^{-1}$. See [ $[\underline{6}$ and its references for some hints for a wide range of applications of this metric in different parts of modern analysis. 
In the paper [6] the second author determined the structure of all surjective Thompson isometries of the space of all invertible positive elements of the $C^{*}$ algebra $B(H)$ (also see [8], where the case $\operatorname{dim} H=2$ was considered separately). The basic idea of the proof given there was, in nice analogy with the proof of the Mazur-Ulam theorem, to show that those isometries necessarily preserve the geometric mean (in the sense of Ando) of invertible positive operators. After that some recent results by the second author concerning transformations preserving the geometric mean or the Jordan triple product were applied to complete the proof. However, the general results in the paper 2] offer another way to approach the problem, which works also in the setting of general $C^{*}$-algebras. In fact, we have the following statement.

Theorem 9. Let $\mathcal{A}, \mathcal{B}$ be unital $C^{*}$-algebras and $\phi: \mathcal{A}_{+}^{-1} \rightarrow \mathcal{B}_{+}^{-1}$ be a surjective isometry with respect to the Thompson metric. Then

$$
\phi\left(y x^{-1} y\right)=\phi(y)(\phi(x))^{-1} \phi(y)
$$

holds for all $x, y \in \mathcal{A}_{+}^{-1}$.

Proof. The condition $\mathrm{B}(a, b)$ holds for every pair $a, b \in \mathcal{A}_{+}^{-1}$. Indeed, for any $x, y, b \in \mathcal{A}_{+}^{-1}$ the equalities $d_{T}\left(b x^{-1} b, b y^{-1} b\right)=d_{T}\left(x^{-1}, y^{-1}\right)=d_{T}(x, y)$ follow directly from the definition of the Thompson metric and, as we see in the proof of [6. Theorem 1], we also have $d_{T}\left(b x^{-1} b, x\right)=2 d_{T}(x, b)$. It follows that $\mathrm{B}(a, b)$ holds for every pair $a, b \in \mathcal{A}_{+}^{-1}$. Moreover, since $\mathcal{B}_{+}^{-1}$ is 2 -divisible, we infer that the condition $\mathrm{C}_{2}(a, b)$ is also satisfied for every pair $a, b \in \mathcal{B}_{+}^{-1}$. Finally, since $\mathcal{B}_{+}^{-1}$ is 2-torsion free as well, applying Proposition [5 we obtain the statement.

We conclude the paper with a remark. If $\phi: \mathcal{A}_{+}^{-1} \rightarrow \mathcal{B}_{+}^{-1}$ is a surjective Thompson isometry, then by the properties of the Thompson metric one can see that the transformation $\psi(\cdot)=\phi(e)^{-1 / 2} \phi(\cdot) \phi(e)^{-1 / 2}(e$ is the unit in $\mathcal{A}$ ) is also a surjective Thompson isometry which sends unit to unit. Now, from the equality $\psi\left(y x^{-1} y\right)=\psi(y)(\psi(x))^{-1} \psi(y)$ for any $x, y \in \mathcal{A}_{+}^{-1}$, we easily conclude that $\psi(y x y)=\psi(y) \psi(x) \psi(y)$ holds for all $x, y \in \mathcal{A}_{+}^{-1}$ (indeed, insert $y=e$ to see that $\psi\left(x^{-1}\right)=(\psi(x))^{-1}$ and then the desired equality follows). This means that $\psi$ is a bijective so-called Jordan triple map from $\mathcal{A}_{+}^{-1}$ onto $\mathcal{B}_{+}^{-1}$. Such transformations have recently been studied quite extensively in different situations (e.g., we refer to the papers [4, 5] by the second author and their references). Especially the question of automatic additivity of such maps has been investigated. Based on those investigations we believe there is some hope that in the future we can relate the Thompson isometries to the linear Jordan *-isomorphisms ( $C^{*}$-isomorphisms) of the underlying algebras, at least in the case of von Neumann algebras, and hence obtain a substantial generalization of the result in [6].

\section{REFERENCES}

[1] O. Hatori, Algebraic properties of isometries between groups of invertible elements in Banach algebras, J. Math. Anal. Appl. 376 (2011), 84-93. MR2745390

[2] O. Hatori, G. Hirasawa, T. Miura and L. Molnár, Isometries and maps compatible with inverted Jordan triple products on groups, Tokyo J. Math., to appear.

[3] S. Mazur and S. Ulam, Sur les transformations isométriques d'espaces vectoriels normés, C. R. Acad. Sci. Paris 194 (1932), 946-948.

[4] L. Molnár, Non-linear Jordan triple automorphisms of sets of self-adjoint matrices and operators, Studia Math. 173 (2006), 39-48. MR2204461 (2006j:47059) 
[5] L. Molnár, Multiplicative Jordan triple isomorphisms on the self-adjoint elements of von Neumann algebras, Linear Algebra Appl. 419 (2006), 586-600. MR2277989 (2007k:47059)

[6] L. Molnár, Thompson isometries of the space of invertible positive operators, Proc. Amer. Math. Soc. 137 (2009), 3849-3859. MR2529894(2010h:47058)

[7] L. Molnár, Selected Preserver Problems on Algebraic Structures of Linear Operators and on Function Spaces, Lecture Notes in Mathematics, Vol. 1895, Springer, 2007. MR.2267033 (2007g:47056)

[8] L. Molnár and G. Nagy, Thompson isometries on positive operators: the 2-dimensional case, Electronic Jour. Linear Algebra 20 (2010), 79-89. MR2596446 (2011c:46040)

[9] L. Molnár and W. Timmermann, A metric on the space of projections admitting nice isometries, Studia Math. 191 (2009), 271-281. MR2481897 (2009m:47097)

[10] W. Rudin, Fourier Analysis on Groups, Interscience Tracts in Pure and Applied Math., No. 12. Wiley, New York, 1962. MR0152834(27:2808)

[11] A.C. Thompson, On certain contraction mappings in a partially ordered vector space, Proc. Amer. Math. Soc. 14 (1963), 438-443. MR0149237 (26:6727)

[12] J. Väisälä, A proof of the Mazur-Ulam theorem, Amer. Math. Monthly 110 (2003), 633-635. MR2001155 (2004d:46021)

Department of Mathematics, Faculty of Science, Niigata University, Niigata 9502181, JAPAN

E-mail address: hatori@math.sc.niigata-u.ac.jp

Institute of Mathematics, University of Debrecen, P.O. Box 12, H-4010 Debrecen, HUNGARY

E-mail address: molnarl@science.unideb.hu

URL: http://www.math.unideb.hu/ molnarl/ 